\title{
Common and distinct patterns of grey matter alterations in borderline personality disorder and bipolar disorder: voxel-based meta-analysis
}

Hua Yu*, Ya-jing Meng* Xiao-jing Li, Chengcheng Zhang, Sugai Liang, Ming-li Li, Zhe Li, Wanjun Guo, Qiang Wang, Wei Deng, Xiaohong Ma, Jeremy Coid and Tao Li

\section{Background}

Whether borderline personality disorder (BPD) and bipolar disorder are the same or different disorders lacks consistency.

\section{Aims}

To detect whether grey matter volume (GMV) and grey matter density (GMD) alterations show any similarities or differences between BPD and bipolar disorder.

\section{Method}

Web-based publication databases were searched to conduct a meta-analysis of all voxel-based studies that compared BPD or bipolar disorder with healthy controls. We included 13 BPD studies (395 patients with BPD and 415 healthy controls) and 47 bipolar disorder studies (2111 patients with bipolar disorder and 3261 healthy controls). Peak coordinates from clusters with significant group differences were extracted. Effect-size signed differential mapping meta-analysis was performed to analyse peak coordinates of clusters and thresholds $(P<0.005$, uncorrected). Conjunction analyses identified regions in which disorders showed common patterns of volumetric alteration. Correlation analyses were also performed.

\section{Results}

Patients with BPD showed decreased GMV and GMD in the bilateral medial prefrontal cortex network (MPFC), bilateral amygdala and right parahippocampal gyrus; patients with bipolar disorder showed decreased GMV and GMD in the bilateral medial orbital frontal cortex (mOFC), right insula and right thalamus, and increased GMV and GMD in the right putamen. Multi-modal analysis indicated smaller volumes in both disorders in clusters in the right medial orbital frontal cortex. Decreased bilateral MPFC in BPD was partly mediated by patient age. Increased GMV and GMD of the right putamen was positively correlated with Young Mania Rating Scale scores in bipolar disorder.

\section{Conclusions}

Our results show different patterns of GMV and GMD alteration and do not support the hypothesis that bipolar disorder and BPD are on the same affective spectrum.

\section{Declaration of interest}

None.

\section{Keywords}

Bipolar disorder; borderline personality disorder; grey matter; meta-analysis; distinct patterns.

\section{Copyright and usage}

(C) The Royal College of Psychiatrists 2019

\section{Similarities and differences between bipolar and borderline personality disorder}

The relationship between borderline personality disorder (BPD) and bipolar disorder remains controversial. ${ }^{1,2}$ Both are severe mental disorders with a fluctuating course. ${ }^{3}$ Bipolar disorder is characterised by manic, depressive and mixed episodes, with intervals showing varying levels of euthymic remission. ${ }^{4}$ BPD is defined as a long-standing pattern of functioning corresponding to a disorder of personality. ${ }^{5}$ However, longitudinal study shows fluctuation with remission of symptoms, including affective instability. ${ }^{6,7}$ Pervasive problems in affect regulation have been considered the core feature of BPD by some researchers, ${ }^{8-11}$ with rapid switching observed between different symptom profiles of anger, depression and anxiety. ${ }^{8,12}$ Symptom profiles and course of affective disturbance appear to differ. ${ }^{13,14}$

Clinical and community studies have shown high levels of diagnostic co-occurrence of the two disorders. ${ }^{15-17}$ A diagnosis of BPD in young people can precede a diagnosis of bipolar disorder in adulthood. ${ }^{18,19}$ It has also been suggested that BPD and bipolar disorder are alternative expressions of the same disorder, ${ }^{20}$ and that BPD is on a bipolar spectrum of affective disorder, ${ }^{1,2,6,21}$ characterised by ultra-rapid-cycling. ${ }^{5}$ It has been suggested that rapid switching

* These authors contributed equally to this work. and the affective instability of BPD could originate from similar genetic aetiology as bipolar disorder. However, mood-stabilising medication is considered the key treatment intervention for bipolar disorder, whereas psychotherapy is the key treatment for BPD. ${ }^{22,23}$ Furthermore, although the two conditions can co-occur, it is argued that their course, family history and treatment response are very different, and that misdiagnosis can result in patients not receiving appropriate treatment. ${ }^{24}$

\section{Neuroimaging studies of the two disorders}

In the absence of a definitive understanding of the neuropathology underpinning these two disorders, there are currently no clinical biomarkers available to aid diagnosis, clarify the relationship between bipolar disorder and BPD or indicate the most appropriate treatment. Biomarker discovery and optimization are therefore essential. Neuroimaging studies have been used to identify differences between patients with affective disorders and healthy controls in structural magnetic resonance imaging (MRI) analysis. ${ }^{25,26}$ Changes in grey matter volume (GMV) and grey matter density (GMD) in BPD have been most consistently found in the amygdala and hippocampus; ${ }^{27,28}$ brain abnormalities in bipolar disorder are frequently reported within the frontal-striatal-limbic network. ${ }^{17,25}$ However, it is unclear whether changes in brain structure can differentiate BPD from bipolar disorder, or whether common 
abnormalities are present in both disorders. ${ }^{18}$ Only one study has directly compared BPD and bipolar disorder, which found that GMD changes in bipolar disorder were more diffuse and severe than in BPD, but had certain regions of overlap. ${ }^{29}$ To date, small sample sizes, heterogeneity and differences in analytical method (e.g. whole-brain or region-of-interest (ROI) analyses) have resulted in inconsistency. A meta-analysis may provide a more precise evaluation of potential diagnostic biomarkers at the brain level, clarify the uncertain relationship between bipolar disorder and BPD and ultimately improve classification. ${ }^{30}$

The aim of this meta-analysis was to establish the most consistent brain function abnormalities of bipolar disorder and BPD, using all published, whole-brain structural MRI studies that do not bias findings to a priori hypothesised regions. ${ }^{31}$

\section{Method}

\section{Searches and study selection}

We conducted a literature search of PubMed (https://www.ncbi. nlm.nih.gov/pubmed/), EMBASE (www.embase.com), Google Scholar (http://scholar.google.com/) and Science Direct (https:// www.sciencedirect.com/) for voxel-based morphometry (VBM) studies published between 1 January 2003 and 28 February 2018. In addition, we conducted manual searches among the reference sections of all retrieved studies and review articles. ${ }^{32}$ To ensure comprehensiveness, two researchers performed the searches independently, using keywords such as 'borderline personality', 'bipolar/affective/mood disorder', 'mania/hypomania/bipolar depression/', 'neuroimaging', 'mri', 'magnetic resonance', 'brain imaging', 'morphometry' and 'voxel'. We followed Preferred Reporting Items For Systematic Reviews and Meta-analyses (http://www.prisma-statement.org) (Fig. 1) guidelines for metaanalyses of observational studies. ${ }^{33}$

Our inclusion criteria were as follows: (a) the study included patients with BPD versus healthy controls, and patients with bipolar disorder versus healthy controls; (b) the VBM method was used to analyse whole-brain grey matter changes of patients with bipolar disorder and patients with BPD; (c) the study reported peak coordinates of the brain areas as well as the statistical parametric maps, and coordinates were normalised into a stereotaxic standardised space (e.g. the Montreal Neurological Institute (MNI) or Talairach space). ${ }^{34}$ Authors of published reports were contacted by email when required information was not provided.

Our exclusion criteria were as follows: (a) neuroimaging techniques other than MRI whole-brain VBM (using ROI/voxelof-interest methods might result in selection bias); (b) participants aged $<18$ years or $>65$ years (to minimise effects of neurodevelopment and neurodegeneration); (c) patients diagnosed with schizoaffective disorders or any comorbid neurological conditions; (d) review papers or meta-analysis studies; and (e) when $t$ - or $z$-maps were unavailable, consistent statistical thresholds throughout the brain were not used or peak coordinates were not reported. ${ }^{25}$ For samples shared with other studies, the study with the largest sample was

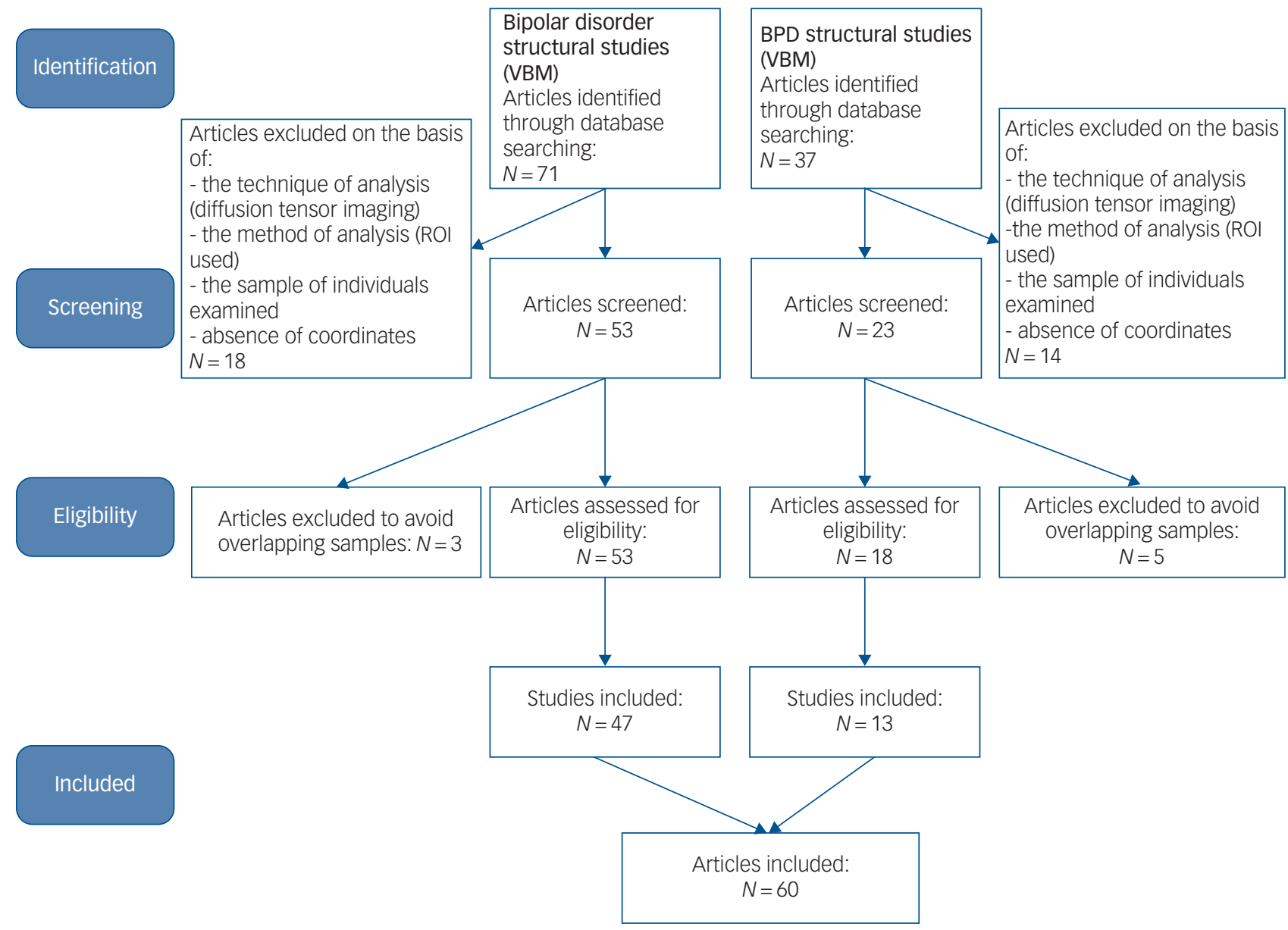

Fig. 1 A Preferred Reporting Items For Systematic Reviews and Meta-analyses (PRISMA) flowchart of the article selection. PRISMA flowchart for the meta-analysis of voxel-based morphometry (VBM) studies in patients with bipolar disorder and borderline personality disorder (BPD). ROI, region of interest. 
included. ${ }^{25}$ Conversely, when the same control group was used in several subgroup comparison, only a combined summary result was included in the meta-analysis. ${ }^{35}$ In the BPD group, we specifically excluded samples with co-occurring bipolar disorder, and vice versa. ${ }^{36}$ For studies that used longitudinal treatment designs, only baseline pre-treatment data were included. Using this approach, we selected a pool of 13 BPD studies and 47 bipolar disorder studies.

\section{Recorded variables and contrasts}

The following variables were recorded from each article: sample size, mean age of participants, gender (male/female), mean illness duration, mean scores of Young Mania Rating Scale (YMRS) and Hamilton Rating Scale for Depression (HRSD) for bipolar disorder, proportion medicated for bipolar disorder, bipolar disorder subtype ( 1 or 2), magnetic field strength and image analysis tools (shown in Supplementary Tables 1 and 2 available at https://doi.org/10.1192/ bjp.2019.44). Coordinates with statistically significant differences were extracted, including direction of alteration (BPD/bipolar disorder $>$ healthy controls, BPD/bipolar disorder $<$ healthy controls). Coordinates in different stereotactic spaces were automatically converted by SDM software and $Z$ - or $P$-values for significant clusters were converted to $t$-statistics by SDM online conversion utilities (https://www.sdmproject.com/utilities/?show=Statistics).

\section{Meta-analysis}

Regional differences in GMV and GMD between patients with BPD or bipolar disorder and healthy controls were analysed using the effect-size version of signed differential mapping (AESSDM; http://www.sdmproject.com/). ${ }^{37}$ Voxel-based meta-analytic methods have been described in detail elsewhere. ${ }^{38}$ In brief, the main features of our method (AES-SDM) included extraction of peak coordinates and effect sizes of grey matter differences between patients with BPD or bipolar disorder and healthy controls from each data-set, ${ }^{2,39}$ recreation of a map of the effect sizes of the differences between patients and controls for each study and a standard random-effects variance-weighted meta-analysis for each voxel. Default AES-SDM kernel size and thresholds were used (full width at half maximum $=20 \mathrm{~mm}$, voxel $P=0.005$, peak height $Z=1$, cluster extent of 10 voxels). ${ }^{3,40}$ We performed the meta-analysis on the weighted mean difference in regional grey matter between patients and healthy controls, weighted by the square root of the sample size of each study, so that studies with large sample sizes contributed more; ${ }^{4,37}$ we confirmed reliability by checking the results; ${ }^{5}$ Jackknife sensitivity analyses were conducted to establish reproducibility of results by iteratively repeating analyses, excluding one data-set each time. ${ }^{41}$

The results replicated in at least $70 \%$ of the studies were reliable. Funnel plots and Egger tests were conducted to identify conflicting studies and publication bias. ${ }^{42} \mathrm{~A} P$-value $<0.05$ was considered significant. ${ }^{43}$ Finally, we used the multi-modal analysis function of the AES-SDM statistical package, which allowed us to perform conjunction analysis. This enabled us to compare abnormalities between conditions (BPD versus bipolar disorder) based on evaluation of effect sizes to identify regions where both patient groups showed shared differences with respect to controls, as well as taking into account errors in the estimation of magnitude of these differences. ${ }^{25}$

\section{Meta-regression analysis}

The potential effect of several relevant sociodemographic and clinical variables were examined by linear regression, weighted by the square root of the sample size and restricted to only predict possible SDM values. ${ }^{41}$ For bipolar disorder, we used depressive symptoms, manic symptoms, illness duration, mean patient age and female patient percentage. Given the relatively small number of studies, we set the cut-off value for inclusion of potential confounders in meta-regressions to $\geq 20$ studies for bipolar disorder to minimise occurrence of false positive values. ${ }^{44}$ For BPD, we used mean patient age and female patient percentage. We also examined effects of magnetic field strength and image smoothing level for both conditions. Studies that did not report these measures were excluded from analyses. ${ }^{25}$

\section{Results}

\section{General information for each sample group}

The 13 structural MRI studies on BPD included 395 patients with BPD and 415 healthy controls (Supplementary Table 1). Their mean age was 29.1 years, $336(75.3 \%)$ were female, 63 (15.95\%) had co-occurring major depressive disorder and 94 (23.80\%) had post-traumatic stress disorder. The 47 bipolar disorder studies included 2111 patients and 3261 healthy controls (Supplementary Table 2). Their mean age was 34.4 years, 1119 (53.01\%) were female, 24 studies reported YMRS scores with mean score of 8.0 and 25 studies reported HRSD scores with mean scores of 8.63 . The bipolar disorder cohorts included 1690 (80.06\%) patients with bipolar disorder type 1, 252 (11.94\%) patients with bipolar disorder type 2 and $176(8 \%)$ patients with other bipolar disorder types. The mean illness duration for bipolar disorder was 14.36 years (30 studies reported). Patients with BPD were significantly younger than patients with bipolar disorder (omnibus test $P<0.001,5.32$ years) and there were more females patients with BPD (omnibus test $P<0.001,23 \%$ female).

\section{Meta-analysis}

\section{BPD versus healthy controls}

Patients with BPD showed significantly decreased GMV and GMD in the bilateral medial prefrontal cortex (mPFC), medial orbital frontal cortex (OFC), bilateral anterior cingulate cortex (ACC), bilateral amygdale and right parahippocampal gyrus compared with healthy controls. They also showed increased GMV and GMD in the bilateral praecuneus, right medium / paracingulate gyrus (Brodmann area (BA) 23) and posterior cingulate gyrus (BA 23) compared with healthy controls (Table 1 and Fig. 2a). Jackknife sensitivity analyses revealed that observed deficits in bilateral posterior cingulate gyrus, bilateral $\mathrm{mPFC}$, medial $\mathrm{OFC}$ and ACC were highly robust and were replicable in all 13 studies. Deficits in bilateral amygdala and right parahippocampal gyrus were replicable in 10 out of 13 studies.

Meta-regression analyses revealed that smaller volumes relative to controls were associated with increasing patient age in the right orbital frontal gyrus (OFC) (Fig. 2b; peak $\mathrm{MNI}=-6,34,-14$; $Z=-3.526 ; P<0.001 ; 1401$ voxels). When examining the methodological variables in the BPD group, studies using higher field strength scanners and higher full width at half maximum showed greater GMV and GMD relative to controls in the right praecuneus (peak $\mathrm{MNI}=12,-38,33 ; Z=1.352 ; P<0.001 ; 189$ voxels; peak $\mathrm{MNI}=6,-58,34 ; Z=1.687 ; P<0.001 ; 541$ voxels).

\section{Bipolar disorder versus healthy controls}

Several reduced GMD and GMV clusters were identified in patients with bipolar disorder compared with healthy controls, including decreased GMV and GMD in the bilateral medial OFC, right insula and right thalamus, whereas increased GMV and GMD was found in the right putamen (Table 1 and Fig. 2c). Whole- 


\begin{tabular}{|c|c|c|c|c|c|c|c|c|}
\hline & $\begin{array}{l}\text { MNI } \\
\text { coordinates }\end{array}$ & Z & $\begin{array}{c}P \\
\text { value }\end{array}$ & $\begin{array}{l}\text { Bonferroni- } \\
\text { corrected } \\
P \text { value* }\end{array}$ & Voxels & Clusters & $\begin{array}{l}\text { Jackknife } \\
\text { sensitivity } \\
\text { analysis }\end{array}$ & $\begin{array}{l}\text { Egger test } \\
P \text { value }\end{array}$ \\
\hline $\begin{array}{l}\text { BPD }>\text { healthy } \\
\text { controls }\end{array}$ & $6,-48,34$ & 1.511 & $<0.001$ & $<0.001$ & 1219 & $\begin{array}{l}\text { Right praecuneus, left praecuneus, right median } \\
\text { cingulate/ paracingulate gyri, left posterior } \\
\text { cingulate gyrus, right posterior cingulate } \\
\text { gyrus }\end{array}$ & 12 in 13 & 0.831 \\
\hline \multirow[t]{3}{*}{$\begin{array}{l}\mathrm{BPD}<\text { healthy } \\
\text { controls }\end{array}$} & $-4,32,-18$ & -2.351 & $<0.001$ & 0.001 & 1287 & $\begin{array}{l}\text { Medial orbital frontal gyrus, anterior cingulated } \\
\text { cortex }\end{array}$ & 11 in 13 & 0.825 \\
\hline & $-28,0,-18$ & -1.964 & 0.001 & 0.015 & 298 & Left amygdala & 9 in 13 & 0.070 \\
\hline & $24,0,-20$ & -1.910 & 0.001 & 0.020 & 252 & Right amygdala, right parahippocampal gyrus & 9 in 13 & 0.111 \\
\hline \multirow{2}{*}{$\begin{array}{l}\text { Bipolar } \\
\text { disorder < } \\
\text { healthy } \\
\text { controls }\end{array}$} & $6,-10,4$ & -2.074 & $<0.001$ & 0.028 & 445 & Right thalamus & 47 in 47 & 0.709 \\
\hline & $\begin{array}{l}6,50,-12 \\
48,2,2\end{array}$ & $\begin{array}{l}-2.347 \\
-2554\end{array}$ & $<0.001$ & $\begin{array}{l}0.014 \\
0.008\end{array}$ & 263 & Medial orbital frontal gyrus, BA 11 & 47 in 47 & 0.965 \\
\hline $\begin{array}{l}\text { Bipolar } \\
\text { disorder }> \\
\text { healthy } \\
\text { controls }\end{array}$ & $30,2,0$ & 1.097 & $<0.001$ & 0.001 & 572 & Right putamen & 47 in 47 & 0.886 \\
\hline
\end{tabular}

brain jackknife sensitivity analysis revealed that the GMV and GMV decrease in the aforementioned brain areas was highly replicable. This finding was observed in all 47 studies and analyses. Metaregression analyses revealed that higher YMRS scores were associated with greater GMV and GMD volumes compared with controls in the right putamen (Fig. 2e; peak $\mathrm{MNI}=32,-8,6 ; Z=$ $2.966 ; P<0.001 ; 684$ voxels). We also found smaller volumes in the right $\mathrm{OFC}$ relative to controls were associated with an increasing percentage of female patients (Fig. 2d; peak $\mathrm{MNI}=6,62,-12$; $Z=-2.93 ; P<0.001 ; 106$ voxels). We found no significant associations between HRSD scores, patient age, mood state, lithium use and methodological variables, and abnormal brain findings in bipolar disorder.

\section{BPD versus bipolar disorder}

Conjunction analysis indicated that brain regions in the right medial OFC showed smaller volume compared with controls in both conditions (Fig. 2f; peak $\mathrm{MNI}=0,34,-20 ; P<0.001 ; 417$ voxels). No regions showed greater volume. Because of small sample sizes directly comparing patients with bipolar disorder and patients with $\mathrm{BPD},{ }^{29}$ we could not contrast the BPD and bipolar disorder group in subgroup analyses.

\section{Analyses of publication bias}

Egger tests for publication bias were not significant with respect to grey matter differences in all study results. All $P$ values were $>0.05$ (Table 1).

\section{Discussion}

To our knowledge, this study is the first voxel-based meta-analysis to investigate differences between individuals with BPD and individuals with bipolar disorder in neuroimaging studies across the entire brain. We compared results to identify both common and different patterns of GMV alterations. We found that the two conditions were substantially different and shared similar patterns of lower volume only in the right medial OFC. However, decreased volume of OFC was partly mediated by patient age in the BPD group. Decreased GMD and GMV of the bilateral amygdala and right parahippocampal gyrus were consistently found in BPD. ${ }^{28,45}$ In contrast, the right insula, right thalamus and right putamen were differently affected in bipolar disorder. Our results indicate that the two disorders are associated with spatially distinct patterns of brain structure.

\section{Overview of emotion dysregulation theory in BPD versus bipolar disorder}

As emotion dysregulation is a core feature of both bipolar disorder and BPD, it can make these two disorders often indistinguishable. ${ }^{46}$ However, differing emotion regulation profiles were observed between the disorders, with emotional dysregulation in those with BPD generally considered to be reactive and precipitated by stressors or interpersonal difficulties, ${ }^{47}$ possibly reflecting behavioural sensitisation (in which repeated exposure to a particular stressor, such as ongoing abuse or neglect, evokes strong reactions). ${ }^{48}$ In bipolar disorder, emotion dysregulation is considered to be more autonomous and internally driven, and also less reactive to psychosocial cues. ${ }^{47}$ Although cognitive and emotion domains are typically studied independently, basic research and emergent findings in bipolar disorder suggest that there are important ties between cognitive deficits and the emotion disturbances observed in bipolar disorder. ${ }^{49}$ A large body of behavioural and neural studies suggests that effective emotion regulation rests on strong executive function, and particularly inhibition. ${ }^{50}$ In accordance with previous BPD and bipolar disorder literature review, our structural neuroimaging results are quite in consistent with the different emotion regulation strategies described above, and we discuss them in detail below.

\section{BPD-specific brain volume abnormality}

Disturbed interpersonal relationships and affect regulation are fundamental aspects of BPD. ${ }^{51}$ The amygdala plays important roles in modulating vigilance and generating negative emotional states and is often abnormally reactive in BPD. ${ }^{52}$ Compared with healthy controls, patients with BPD showed greater amygdala activation, particularly in response to repeated emotional stimuli, and a prolonged return to baseline for overall blood oxygen level-dependent response averaged across all pictures. ${ }^{53}$ The decreased amygdala 
(a)

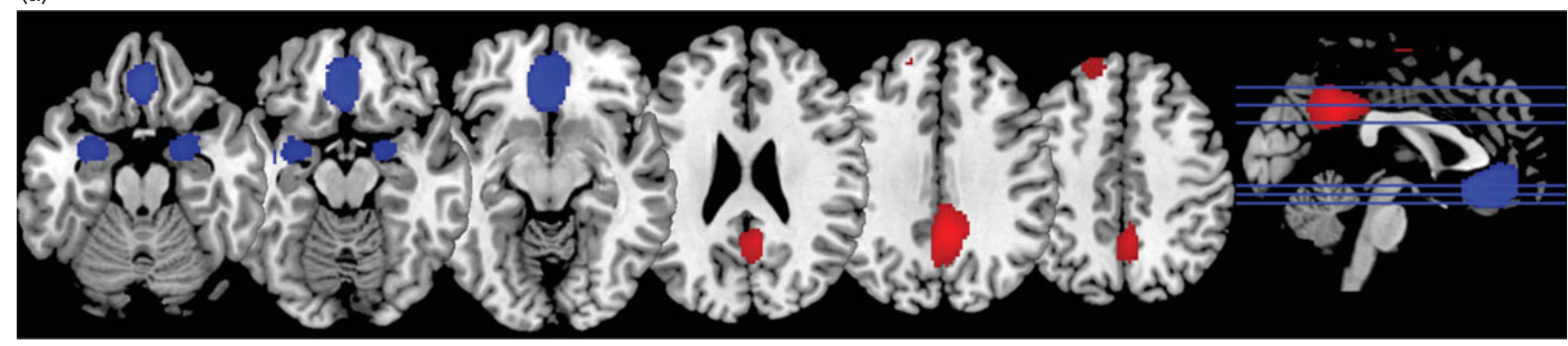

(b)
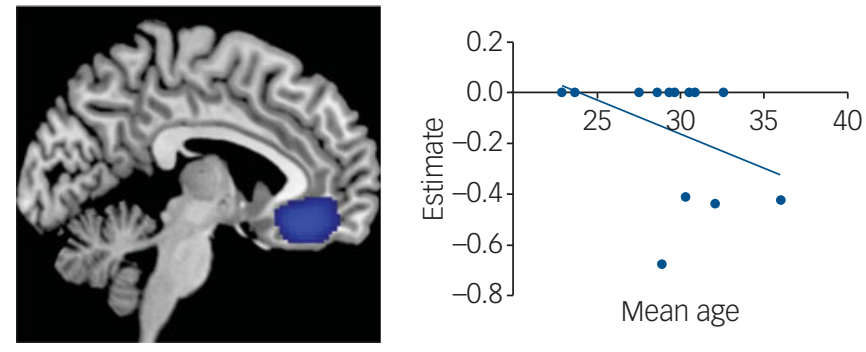

(c)

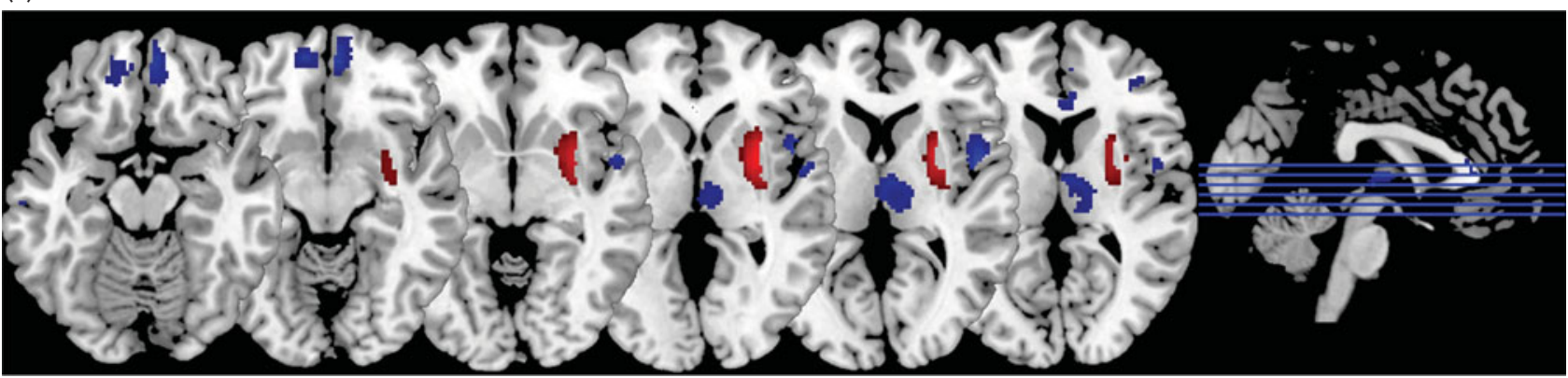

(d)

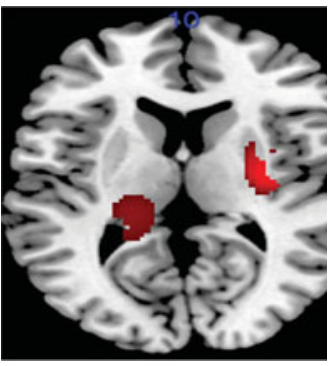

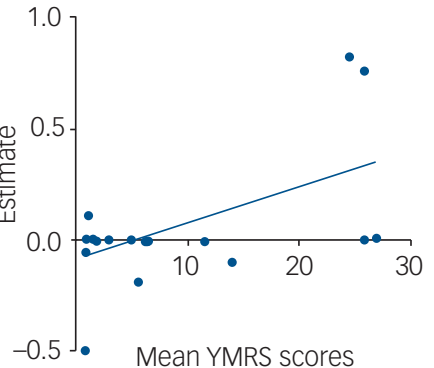

Mean YMRS scores (e)

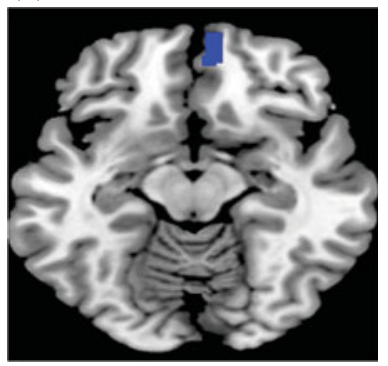

(f)

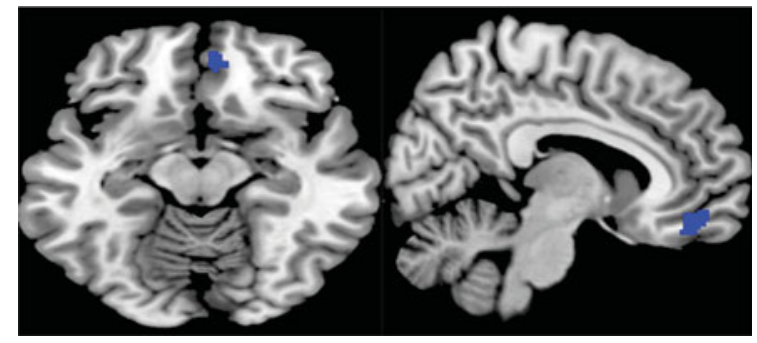

Fig. 2 Meta-analysis results. (a) Results of borderline personality disorder (BPD) meta-analysis. (b) Results of meta-regression with age in BPD (c) Results of bipolar disorder meta-analysis. (d) Results of meta-regressions with Young Manic Rating Scale (YMRS) scores in bipolar disorder. (e) Results of meta-regression with gender in bipolar disorder. (f) Results of the conjunction analysis showing regions with similar volumetric alterations in both conditions. Blue represents lower volume in both conditions relative to controls; red represents greater volume relative to controls or negative relationships with regressors. In meta-regression plots, point size represents study weights. All images are shown in neurological convention; the left of the image corresponds to left of the brain. Effect sizes represent effect sizes at the peak of the cluster. 
volume here we found might be the structural basis for the functional abnormalities as previously described by O’Neill and Frodl. ${ }^{54}$ The adjacent parahippocampal gyrus projects to the hippocampus and the limbic circuit, and plays a role in memory encoding and retrieval, especially with regard to retrieving information about familiarity of scenes. It also plays a role in identifying sarcasm in verbal communication. ${ }^{55}$ Retrieval of specific and positive autobiographical memories, a function of the hippocampus, is impaired in BPD and is related to poor problem-solving. ${ }^{56}$ During an emotional crisis, this associative memory function enables a person to envision positive outcomes based on past experiences. ${ }^{57}$ The subcortical area abnormalities we found in BPD are quite inconsistent with the previous published meta-analysis. In 2012, Ruocco et al ${ }^{45}$ reported that the bilateral volumes of amygdala and hippocampus were modestly reduced in $\mathrm{BPD}$, which cannot be attributed to illness state or comorbid psychopathology.

Studies in healthy controls suggested intact coupling between prefrontal cortex, particularly the ventral prefrontal cortex, and amygdala, the parahippocampal gyrus may be the neural substrate for downregulation of the amygdala in response to aversive stimuli. ${ }^{58}$ The absence of such close coupling in patients with $\mathrm{BPD}$, indicated by the lack of significant correlations between the OFC and amygdala/parahippocampal gyrus, suggests a disconnect between the OFC and limbic areas, which may explain the failure of patients with BPD to downregulate the subcortical areas in response to aversive stimuli. ${ }^{59}$ It is likely that the $\mathrm{mPFC}$ volume deficit is partly mediated by age and that the affected higher-order brain areas are not as pervasive as in bipolar disorder. The less-affected higher-order cortical cortex might explain why patients with BPD benefit from treatments targeting emotion recognition. ${ }^{60}$

\section{Bipolar disorder-related brain volume abnormality}

Our results are consistent with previous studies performed in patients with bipolar disorder and affective disorders. ${ }^{25,61}$ Wise et al reported abnormal GMV changes in $\mathrm{mPFC}$ systems, the insula and limbic areas in bipolar disorder, ${ }^{25}$ and Phillips et al proposed the theory that in mood dysregulation in affective disorders, differences in the brain regions amygdala, insula, ventral striatum, ACC and prefrontal cortex may lead to the aberrant mood states. ${ }^{61}$ The insular cortex has recently become an area of focus in psychiatric research, particularly the anterior insula cortex (AIC). This region is involved in a range of functions, including affective processing and awareness of bodily states. ${ }^{62,63}$ Atypical functioning of this region in affective disorders has been found in functional neuroimaging research and convergent evidence indicates insular abnormalities in bipolar disorder. ${ }^{64,65}$ Our finding of smaller insular volume in patients with bipolar disorder suggests that structural abnormalities are present in the same areas in which altered function has also been identified previously, and may imply impairment of insula functioning, leading to abnormal cognitive and visceral responses to negative emotional stimuli. $^{66}$

The thalamus serves as a relay station within forebrain circuits to the cerebral cortex and to limbic structures. ${ }^{67}$ It has been increasingly implicated in emotional and cognitive processing, particularly via cortico-basal ganglia and cortico-thalamic circuits, and appears to play important roles in executive functions that are commonly impaired in psychotic conditions. ${ }^{68}$ Functional connectivity data have also shown functional connections between the thalamus and anterior insula, ${ }^{69}$ and the connections between the AIC and thalamus suggest that the AIC is involved in modulation of thalamic function. Our results further confirm previous studies reporting that thalamic dysfunction is combined with insula abnormalities, which is associated with aberrant emotional, cognitive and social behaviour in bipolar disorder. ${ }^{70}$ Our meta-analysis showed increased GMD and GMV of the striatum and is positively correlated with YMRS scores. Therefore, increased volume of putamen is very likely a state effect. The results are also consistent with clinical observations that patients with bipolar disorder have enhanced reward intensity. ${ }^{71}$

We also found decreased GMV and GMD of OFC in bipolar disorder. Rodent and primate studies indicate that the OFC is involved in the assessment of emotional significance and reward potential of stimuli, and regulates the amygdala. ${ }^{71,72}$ Abnormalities in this system can cause an inability to assess emotional significance of stimuli, leading to inappropriate emotional responses and impulsive behaviour. ${ }^{73}$ In our results, we found decreased OFC volume in patients with bipolar disorder, which was more severe in female patients, consistent with previous evidence showing that female patients with bipolar disorder report higher levels of both positive and negative affect over time. ${ }^{74}$ Our data partially support the hypothesis that mood disorder represents a decrease in cortical activation and increase in limbic activation, but also highlights a more complex and nuanced picture that differs across mood states/traits and cortical subregions.

\section{Common affected medial OFC in BPD and bipolar disorder}

Our finding of OFC deficits in both clinical groups may indicate that these changes are not BPD-specific but rather imply a general biological vulnerability to the development of psychiatric disturbances. ${ }^{75}$ For instance, deficits in OFC areas are shared with other psychiatric disorders like depression ${ }^{72,76}$ and addiction disorders. $^{77,78}$ Thus, it may be hypothesised that BPD could be a frontal-deficit spectrum disease that shares frontal deficits with disorders other than bipolar disorder. Our finding of prefrontal deficits may be less of an endophenotypic pattern for a specific diagnosis, but may instead represent a pattern that reflects dysfunctioning of cognitive abilities (like attention, working memory and declarative memory), which impairs the capacity to control emotions $(129,130)$. However, further longitudinal studies that over the time course of the disease are needed to verify whether the pattern of changes in brain morphology is unique for BPD. Longitudinal development in BPD should then be compared with longitudinal developments in other disorders. ${ }^{75}$

\section{Limitations}

There are several limitations to this study. The first limitation comes from the SDM VBM method, as it was based on peak coordinates and effect sizes from published studies rather than raw statistical brain maps, which may result in less accurate findings. ${ }^{31}$ To date, there have been few VBM studies directly comparing bipolar disorder and BPD, making it difficult to draw definitive conclusions on similarities and differences. In addition, by using the SDM VBM method we cannot exclude the possibility that our finding of abnormal subcortical areas (i.e. abnormal parahippocampal gyrus and amygdala volumes in BPD) but no differences in amygdala volume in bipolar disorder may be due to the edge effects or reduced sensitivity of whole-brain VBM analyses in small regions, such as the hippocampus and amygdala. ${ }^{79}$ Manual segmentation remains the gold standard for analysing subcortical structures like the hippocampus and the amygdala. However, this method has limitations, such as obtaining each ROI in the native space without image transformation, as well as being operator-dependent and highly time-consuming. ${ }^{80}$ Other popular method-automatic segmentation, such as the ROI-based approach by FreeSurfer, is increasingly accepted. Even for small regions, entirely automated 
use of FreeSurfer is acceptable in large data-sets. However, the approach used in this meta-analysis, although indirectly comparing studies' effect sizes, offers the most viable option that allows for conclusions generalisable beyond individual studies. Furthermore, given the robustness of the meta-analytic method, our results provide a summary of the most reliable differences observed between bipolar disorder and BPD.

The second limitation is the lack of heterogeneity analysis across the patient populations. Clinical differentiation is seemingly more difficult in differentiating BPD from the bipolar disorder type 2 subtype. ${ }^{81}$ However, within the studies included in this analysis, only one included the bipolar disorder type 2 samples in their analysis, ${ }^{82}$ so we cannot perform a subgroup analysis directly by dividing the bipolar disorder group into type 1 and 2 subtypes. Furthermore, the prevalence of psychosis in BPD and bipolar disorder groups could significantly contribute to the differences reported in this study because of the comorbidity between psychosis and both groups. ${ }^{83}$ Further studies should be performed using subgroup analysis considering the issues of comorbidities.

Third, we cannot determine causality from these results because all studies were cross-sectional group comparisons. It is unclear whether these alterations are part of the genetic pathogenesis of these disorders or a consequence of illness. ${ }^{25}$ In addition, given that psychotropic medications and psychotherapy can have demonstrable effects on brain structure, ${ }^{84,85}$ it is difficult to be certain that results are not entirely independent from treatment status. To solve these issues, longitudinal and clinical trials focusing on treatment effects are needed in future studies.

Finally, several studies reported GMD rather than volume. The mean GMD is derived from the percentage of absolute GMV divided by total brain volume. This might result in different locations of deficit areas from results achieved by VBM measurements of GMV. ${ }^{86}$

In conclusion, BPD and bipolar disorder show distinct patterns of GMV abnormalities in a number of regions. Differences were predominantly observed in the amygdala and parahippocampal gyrus in BPD and the cortical-thalamic-striatal circuit in bipolar disorder. Although there is significant heterogeneity within these results, this was partially explained by clinical and demographic differences in clinical samples. These findings suggest new targets for neuroanatomical diagnostic biomarkers. They do not suggest that BPD falls within the bipolar spectrum.

\footnotetext{
Hua Yu, MD (D), Associate Researcher, Mental Health Center, West China Hospital of Sichuan University; Psychiatric Laboratory, State Key Laboratory of Biotherapy, West China Hospital of Sichuan University; and Brain Research Center, West China Hospital of Sichuan University, China; Ya-jing Meng, MD, Associate Researcher, Mental Health Center, West China Hospital of Sichuan University; Psychiatric Laboratory, State Key Laboratory of Biotherapy, West China Hospital of Sichuan University; and Brain Research Center, West China Hospital of Sichuan University, China; Xiao-jing Li, MD, Associate Researcher, Mental Health Center, West China Hospital of Sichuan University; Psychiatric Laboratory, State Key Laboratory of Biotherapy, West China Hospital of Sichuan University; and Brain Research Center, West China Hospital of Sichuan University, China; Chengcheng Zhang, MD, Associate Researcher, Mental Health Center, West China Hospital of Sichuan University; Psychiatric Laboratory, State Key Laboratory of Biotherapy, West China Hospital of Sichuan University; and Brain Research Center, West China Hospital of Sichuan University, China; Sugai Liang, MD, Associate Researcher, Mental Health Center, West China Hospital of Sichuan University; Psychiatric Laboratory, State Key Laboratory of Biotherapy, West China Hospital of Sichuan University; and Brain Research Center, West China Hospital of Sichuan University, China; Ming-li Li, MD, Mental Health Center, West China Hospital of Sichuan University; Psychiatric Laboratory, State Key Laboratory of Biotherapy, West China Hospital of Sichuan University; and Brain Rental the State Key Laboratory of Biotherapy, West China Hospital of Sichuan University; and Brain Research Center, West China Hospital of Sichuan University, China; Wanjun Guo, MD, Lecturer, Mental Health Center, West China Hospital of Sichuan University; Psychiatric Laboratory, State Key Laboratory of Biotherapy, West China Hospital of Sichuan University; and Brain Research Center, West China Hospital of Sichuan University, China; Qiang Wang, MD, Lecturer, Mental Health Center, West China Hospital of Sichuan University; Psychiatric Laboratory, State Key Laboratory of Biotherapy, West China University; Psychiatric Laboratory, State Key Laboratory of Biotherapy, West China
Hospital of Sichuan University; and Brain Research Center, West China Hospital of Sichuan University, China; Wei Deng, MD, Lecturer, Mental Health Center, West China Hospital of Sichuan University; Psychiatric Laboratory, State Key Laboratory of
}

Biotherapy, West China Hospital of Sichuan University; and Brain Research Center, West China Hospital of Sichuan University, China; Xiaohong Ma, MD, Researcher, Mental Health Center, West China Hospital of Sichuan University; Psychiatric Laboratory, State Key Laboratory of Biotherapy, West China Hospital of Sichuan University; and Brain Research Center, West China Hospital of Sichuan University, China; Jeremy Coid, MD, Researcher, Mental Health Center, West China Hospital of Sichuan University; Psychiatric Laboratory, State Key Laboratory of Biotherapy, West China Hospital of Sichuan University; and Brain Research Center, West China Hospital of Sichuan University, China; Tao Li, MD, Researcher, Mental Health Center, West China Hospital of Sichuan University; Psychiatric Laboratory, State Key Laboratory of Biotherapy, West China Hospital of Sichuan University; and Brain Research Center, West China Hospital of Sichuan University, China

Correspondence: Dr Tao Li, Mental Health Center, West China Hospital, SIchuan University, 28th South Dianxin Road, Wuhou District, Chnegdu, Sichuan 610041, China. Email: litaohx@scu.edu.cn

First received 27 Jul 2018, final revision 22 Jan 2019, accepted 25 Jan 2019

\section{Supplementary material}

Supplementary material is available online at https://doi.org/10.1192/bjp.2019.44.

\section{Acknowledgements}

This work was partly funded by the National Key Research and Development Program of the Ministry of Science and Technology of China (grant 2016 YFC0904300 to T.L.), the National Nature Science Foundation of China Key Project (grants 91332205, 81630030 and 81130024 to T.L.), the National Nature Science Foundation of China Project (grant 81601172 to Y.M., 81501174 to M.L.L. and 81681571305 to W.G.), the National Natural Science Foundation of China/Research Grants Council of Hong Kong Joint Research Scheme (grant 81461168029 to T.L) and the 1.3.5 Project for disciplines of excellence, West China Hospital of Sichuan University (grants ZY2016103 and ZY2016203 to T.L.).

\section{References}

1 Akiskal HS. Subaffective disorders: dysthymic, cyclothymic and bipolar II disorders in the 'borderline' realm. Psychiatr Clin North Ame 1981; 4: 25-46.

2 Benazzi F. A relationship between bipolar II disorder and borderline personality disorder? Prog Neuropsychopharmacol Biol Psychiatry 2008; 32: 1022-9.

3 American Psychiatric Association (APA). Diagnostic and Statistical Manual of Mental Disorders (DSM). APA, 2013.

4 Vieta E, Berk M, Schulze TG, Carvalho AF, Suppes T, Calabrese JR, et al. Bipolar disorders. Nat Rev Dis Primers 2018; 4: 18008.

5 Akiskal HS. Demystifying borderline personality: critique of the concept and unorthodox reflections on its natural kinship with the bipolar spectrum. Acta Psychiatr Scand 2004; 110: 401-7.

6 Akiskal HS, Chen SE, Davis GC, Puzantian VR, Kashgarian M, Bolinger JM. Borderline: an adjective in search of a noun. J Clin Psychiatry 1985; 46: 41-8.

7 Zanarini MC, Vujanovic AA, Parachini EA, Boulanger JL, Frankenburg FR, Hennen J. A screening measure for BPD: the McLean Screening Instrument for Borderline Personality Disorder (MSI-BPD). J Pers Disord 2003; 17(6): 568-73.

8 Coid JW. An affective syndrome in psychopaths with borderline personality disorder? Br J Psychiatry 1993; 162: 641-50.

9 Corrigan FM, Davidson A, Heard $\mathrm{H}$. The role of dysregulated amygdalic emotion in borderline personality disorder. Med Hypotheses 2000; 54: 574-9.

10 Glenn CR, Klonsky ED. Emotion dysregulation as a core feature of borderline personality disorder. J Pers Disord 2009; 23(1): 20-8.

11 Linehan MM. Cognitive Behavioral Therapy of Borderline Personality Disorder. Guilford Press, 1993

12 Reisch T, Ebner-Priemer UW, Tschacher W, Bohus M, Linehan MM. Sequences of emotions in patients with borderline personality disorder. Acta Psychiatr Scand 2010; 118: 42-8.

13 Henry C, Mitropoulou V, New AS, Koenigsberg HW, Silverman J, Siever LJ. Affective instability and impulsivity in borderline personality and bipolar II disorders: similarities and differences. J Psychiatr Res 2001; 35(6): 307-12.

14 Koenigsberg HW. Affective instability: toward an integration of neuroscience and psychological perspectives. J Pers Disord 2010; 24(1): 60-82.

15 Gunderson JG, Weinberg I, Daversa MT, Kueppenbender KD, Zanarini MC Shea MT, et al. Descriptive and longitudinal observations on the relationship of borderline personality disorder and bipolar disorder. Am J Psychiatry 2006; 163: $1173-8$.

16 Zimmerman M, Morgan TA. The relationship between borderline personality disorder and bipolar disorder. Dialogues Clin Neurosci 2013; 15: 155-69. 
17 Pereira LP, Köhler CA, De Sousa RT, Solmi M, De Freitas BP, Fornaro M, et al. The relationship between genetic risk variants with brain structure and function in bipolar disorder : a systematic review of genetic-neuroimaging studies. Neurosci Biobehav Rev 2017; 79(April): 87-109.

18 McDermid J, Sareen J, El-Gabalawy R, Pagura J, Spiwak R, Enns MW. Co-morbidity of bipolar disorder and borderline personality disorder: findings from the National Epidemiologic Survey on Alcohol and Related Conditions. Compr Psychiatry 2015; 58: 18-28.

19 Yen S, Frazier E, Hower H, Weinstock LM, Topor DR, Hunt J, et al. Borderline personality disorder in transition age youth with bipolar disorder. Acta Psychiatr Scand 2015; 132: 270-80.

20 de la Rosa I, Oquendo MA, García G, Stanley B, González-Pinto A, Liu S-M, et al. Determining if borderline personality disorder and bipolar disorder are alternative expressions of the same disorder: results from the national epidemiologic survey on alcohol and related conditions. J Clin Psychiatry 2017; 78(8): e994-9.

21 Walsh MA, DeGeorge DP, Barrantes-Vidal N, Kwapil TR. A 3-year longitudinal study of risk for bipolar spectrum psychopathology. J Abnorm Psychol 2015; 124: $486-97$

22 Ruggero CJ, Zimmerman M, Chelminski I, Young D. Borderline personality disorder and the misdiagnosis of bipolar disorder. Psychiatry 2011; 44: 405-8.

23 Gunderson JG, Phillips A. A current view of the interface between borderline personality disorder and depression. Am J Psychiatry 1991; 148: 967-75.

24 Paris J, Black DW. Borderline personality disorder and bipolar disorder. J NerV Ment Dis 2015; 203: 3-7.

25 Wise T, Radua J, Via E, Cardoner N, Abe O, Adams TM, et al. Common and distinct patterns of grey-matter volume alteration in major depression and bipolar disorder: evidence from voxel-based meta-analysis. Mol Psychiatry 2017; 22(10): 1455-63.

26 Ganzola R, Duchesne S. Voxel-based morphometry meta-analysis of gray and white matter finds significant areas of differences in bipolar patients from healthy controls. Bipolar Disord 2017; 19: 74-83.

27 Nunes PM, Wenzel A, Borges KT, Porto CR, Caminha RM, de Oliveira IR. Volumes of the hippocampus and amygdala in patients with borderline personality disorder: a meta-analysis. J Pers Disord 2009; 23(4): 333-45.

28 Yang $X$, Hu L, Zeng J, Tan Y, Cheng B. Default mode network and frontolimbic gray matter abnormalities in patients with borderline personality disorder: a voxel-based meta-analysis. Sci Rep 2016; 6: 34247

29 Rossi R, Pievani M, Lorenzi M, Boccardi M, Beneduce R, Bignotti S, et al. Structural brain features of borderline personality and bipolar disorders. Psychiatry Res Neuroimaging 2013; 213(2): 83-91.

30 Kempton MJ, Salvador Z, Munafò MR, Geddes JR, Simmons A, Frangou S, et al. Structural neuroimaging studies in major depressive disorder: meta-analysis and comparison with bipolar disorder. Arch Gen Psychiatry 2011; 68(7): 675-90.

31 Lim L, Radua J, Rubia K. Gray matter abnormalities in childhood maltreatment: a voxel-wise meta-analysis. Am J Psychiatry 2014; 8: 854-63.

32 Norman $\sqcup$, Carlisi C, Lukito S, Hart H, Mataix-Cols D, Radua J, et al. Structura and functional brain abnormalities in attention-deficit/hyperactivity disorder and obsessive-compulsive disorder: a comparative meta-analysis. JAMA Psychiatry 2016; 73(8): 815-25.

33 Modinos G, Costafreda SG, van Tol M-J, McGuire PK, Aleman A, Allen P. Neuroanatomy of auditory verbal hallucinations in schizophrenia: a quantitative meta-analysis of voxel-based morphometry studies. Cortex 2013; 49(4): 1046-55.

34 Bora $\mathrm{E}$, Fornito $\mathrm{A}$, Yücel M, Pantelis $\mathrm{C}$. Voxelwise meta-analysis of gray matter abnormalities in bipolar disorder. Biol Psychiatry 2010; 67(11): 1097-105.

35 Schulze L, Schmahl C, Niedtfeld I. Neural correlates of disturbed emotion processing in borderline personality disorder: a multimodal meta-analysis. Biol Psychiatry 2016; 79(2): 97-106.

36 Bruno SD, Barker GJ, Cercignani M, Symms M, Ron MA. A study of bipolar disorder using magnetization transfer imaging and voxel-based morphometry. Brain 2004; 127(11): 2433-40.

37 Radua J, Rubia K, Canales-Rodríguez EJ, Pomarol-Clotet E, Fusar-Poli P, MataixCols D. Anisotropic kernels for coordinate-based meta-analyses of neuroimaging studies. Front Psychiatry 2014; 5: 13.

38 Nakao T, Radua J, Rubia K, Mataix-Cols D. Gray matter volume abnormalities in ADHD: voxel-based meta-analysis exploring the effects of age and stimulant medication. Am J Psychiatry 2011; 168(11): 1154-63.

39 Hart H, Radua J, Nakao T, Mataix-Cols D, Rubia K. Meta-analysis of functional magnetic resonance imaging studies of inhibition and attention in attentiondeficit/hyperactivity disorder: exploring task-specific, stimulant medication, and age effects. JAMA Psychiatry 2013; 70(2): 185-98.

40 Radua J, Borgwardt S, Crescini A, Mataix-Cols D, Meyer-Lindenberg A, McGuire PK, et al. Multimodal meta-analysis of structural and functional brain changes in first episode psychosis and the effects of antipsychotic medication. Neurosci Biobehav Rev 2012; 36(10): 2325-33.

41 Radua J, Mataix-Cols D. Voxel-wise meta-analysis of grey matter changes in obsessive-compulsive disorder. Br J Psychiatry 2009; 195(5): 393-402.

42 Carlisi CO, Norman $\sqcup$, Lukito SS, Radua J, Mataix-cols D, Rubia K. Archival report comparative multimodal meta-analysis of structural and functional brain abnormalities in autism spectrum disorder and obsessive-compulsive disorder. Biol Psychiatry 2017; 82: 83-102.

43 Pan $\mathrm{P}$, Zhan $\mathrm{H}$, Xia M, Zhang Y, Guan $\mathrm{D}$, Xu Y. Aberrant regional homogeneity in Parkinson's disease: a voxel-wise meta-analysis of resting-state functional magnetic resonance imaging studies. Neurosci Biobehav Rev 2017; 72 : 223-31.

44 Higgins JP, Thompson SG. Controlling the risk of spurious findings from metaregression. Stat Med 2004; 23(11): 1663-82.

45 Ruocco AC, Amirthavasagam S, Zakzanis KK. Amygdala and hippocampal volume reductions as candidate endophenotypes for borderline personality disorder: a meta-analysis of magnetic resonance imaging studies. Psychiatry Res Neuroimaging 2012; 201(3): 245-52.

46 Coulston CM, Tanious M, Mulder RT, Porter RJ, Malhi GS. Bordering on bipolar: the overlap between borderline personality and bipolarity. Aust New Zeal $\rfloor$ Psychiatry 2012; 46(6): 506-21.

47 Renaud S, Corbalan F, Beaulieu S. Differential diagnosis of bipolar affective disorder type II and borderline personality disorder: analysis of the affective dimension. Compr Psychiatry 2012; 53(7): 952-61.

48 Post RM, Weiss SRB. Sensitization and kindling phenomena in mood, anxiety, and obsessive-compulsive disorders: the role of serotonergic mechanisms in illness progression. Biol Psychiatry 1998; 44(3): 193-206.

49 Lima IMM, Peckham AD, Johnson SL. Cognitive deficits in bipolar disorders: implications for emotion. Clin Psychol Rev 2018; 59: 126-36.

50 Green MJ, Cahill CM, Malhi GS. The cognitive and neurophysiological basis of emotion dysregulation in bipolar disorder. J Affect Disord 2007; 103(1-3): 29-42.

51 Zanarini MC, Frankenburg FR, Reich DB, Silk KR, Hudson Jl, McSweeney LB. The subsyndromal phenomenology of borderline personality disorder: a 10-year follow-up study. Am J Psychiatry 2007; 164: 929-35.

52 Niedtfeld I, Schulze L, Krause-Utz A, Demirakca T, Bohus M, Schmahl C. Voxelbased morphometry in women with borderline personality disorder with and without comorbid posttraumatic stress disorder. PLOS ONE 2013; 8(6): e65824

53 Donegan NH, Sanislow CA, Blumberg HP, Fulbright RK, Lacadie C, Skudlarski P, et al. Amygdala hyperreactivity in borderline personality disorder: implications for emotional dysregulation. Biol Psychiatry 2003; 54(11): 1284-93.

$54 \mathrm{O}^{\prime}$ Neill A, Frodl T. Brain structure and function in borderline personality disorder. Brain Struct Funct 2012; 217(4): 767-82.

55 Rankin KP, Salazar A, Gorno-Tempini ML, Sollberger M, Wilson SM, Pavlic D, et al. Detecting sarcasm from paralinguistic cues: anatomic and cognitive correlates in neurodegenerative disease. Neuroimage 2009: 2005-15.

56 Mark J, Broadbent K. Autobiographical memory in suicide attempters. J Abnor Psychol 1986; 95: 144-9.

57 Soloff PH, Pruitt P, Sharma M, Radwan J, White R, Diwadkar VA. Structural brain abnormalities and suicidal behavior in borderline personality disorder. $J$ Psychiatr Res 2013; 46: 516-25.

58 Tebartz van Elst L, Hesslinger B, Thiel T, Geiger E, Haegele $K$, Lemieux L, et al. Frontolimbic brain abnormalities in patients with borderline personality disorder: a volumetric magnetic resonance imaging study. Biol Psychiatry 2003; 54: 163-71.

59 New AS, Hazlett EA, Buchsbaum MS, Goodman M, Mitelman SA, Newmark R, et al. Amygdala-prefrontal disconnection in borderline personality disorder. Neuropsychopharmacology 2007; 32: 1629-40.

60 Hazlett EA, Zhang J, New AS, Zelmanova Y, Goldstein KE, Haznedar MM, et al. Potentiated amygdala response to repeated emotional pictures in borderline personality disorder. Biol Psychiatry 2012; 72(6): 448-56.

61 Phillips ML, Ladouceur CD, Drevets WC. A neural model of voluntary and automatic emotion regulation: implications for understanding the pathophysiology and neurodevelopment of bipolar disorder. Mol Psychiatry 2008; 13(9): 833-57.

62 Li J, Tang Y, Womer F, Fan G, Zhou Q, Sun W, et al. Two patterns of anterio insular cortex functional connectivity in bipolar disorder and schizophrenia. World J Biol Psychiatry 2018; 19(sup3): S115-S123.

63 Lochhead RA, Parsey R V, Oquendo MA, Mann JJ. Regional brain gray matter volume differences in patients with bipolar disorder as assessed by optimized voxel-based morphometry. Biol Psychiatry 2004; 55(12): 1154-62.

64 Brooks III JO, Wang PW, Bonner JC, Rosen AC, Hoblyn JC, Hill SJ, et al. Decreased prefrontal, anterior cingulate, insula, and ventral striatal metabolism in medication-free depressed outpatients with bipolar disorder. J Psychiatr Res 2009; 43(3): 181-8 
65 Bechdolf A, Wood SJ, Nelson B, Velakoulis D, Yücel M, Takahashi T, et al. Amygdala and insula volumes prior to illness onset in bipolar disorder: a magnetic resonance imaging study. Psychiatry Res Neuroimaging 2012; 201(1): 34-9.

66 Bush G, Luu P, Posner Ml. Cognitive and emotional influences in anterior cingulate cortex. Trends Cogn Sci 2000; 4(6): 215-22.

67 Jones EG. Cortical development and thalamic pathology in schizophrenia. Schizophr Bull 1997; 23(3): 483-501.

68 Womer FY, Wang L, Alpert KI, Smith MJ, Csernansky JG, Barch DM, et al. Basal ganglia and thalamic morphology in schizophrenia and bipolar disorder. Psychiatry Res Neuroimaging 2014; 223(2): 75-83.

69 Chai XJ, Whitfield-Gabrieli S, Shinn AK, Gabrieli JDE, Nieto Castañón A McCarthy JM, et al. Abnormal medial prefrontal cortex resting-state connectivity in bipolar disorder and schizophrenia. Neuropsychopharmacology 2011; 36: 2009-17.

70 Strakowski SM, Delbello MP, Adler CM. The functional neuroanatomy of bipolar disorder: a review of neuroimaging findings. Mol Psychiatry 2012; 10(1): 105-16.

71 Nusslock R, Almeida JR, Forbes EE, Versace A, Frank E, Labarbara EJ, et al. Waiting to win: elevated striatal and orbitofrontal cortical activity during reward anticipation in euthymic bipolar disorder adults. Bipolar Disorder 2012; 14(3): 249-60.

72 Öngür D, Price JL. The organization of networks within the orbital and media prefrontal cortex of rats, monkeys and humans. Cereb Cortex 2000; 10(3): 206-19.

73 Antoniadis D, Samakouri M, Livaditis M. The association of bipolar spectrum disorders and borderline personality disorder. Psychiatr Q 2012; 83(4): 449-65.

74 Diener E, Sandvik E, Larsen RJ. Age and sex effects for emotional intensity. Dev Psychol 1985: 542-6.

75 Brunner R, Henze R, Parzer P, Kramer J, Feigl N, Lutz K, et al. Reduced prefronta and orbitofrontal gray matter in female adolescents with borderline personality disorder: is it disorder specific? Neuroimage 2010; 49(1): 114-20.

76 Murray EA, Wise SP, Drevets WC. Localization of dysfunction in major depressive disorder: prefrontal Cortex and Amygdala. Biol Psychiatry 2011; 69(12): e43-54.

77 Volkow ND, Wang G-J, Ma Y, Fowler JS, Wong C, Ding Y-S, et al. Activation of orbital and medial prefrontal cortex by methylphenidate in cocaine-addicted subjects but not in controls: relevance to addiction. J Neurosci 2005; 25(15): 3932-9.

78 Kantak KM, Yager LM, Brisotti MF. Impact of medial orbital cortex and medial subthalamic nucleus inactivation, individually and together, on the maintenance of cocaine self-administration behavior in rats. Behav Brain Res 2013; 238: 1-9.

79 Bergouignan L, Chupin M, Czechowska Y, Kinkingnéhun S, Lemogne $C$, Le Bastard G, et al. Can voxel based morphometry, manual segmentation and automated segmentation equally detect hippocampal volume differences in acute depression? Neuroimage 2009; 45(1): 29-37.

80 Grimm O, Pohlack S, Cacciaglia R, Winkelmann T, Plichta MM, Demirakca T, et al. Amygdalar and hippocampal volume: a comparison between manual segmentation, Freesurfer and VBM. J Neurosci Methods 2015; 253: 254-61.

81 Fletcher K, Parker G, Bayes A, Paterson A, McClure G. Emotion regulation strategies in bipolar II disorder and borderline personality disorder: differences and relationships with perceived parental style. J Affect Disord 2014; 157 52-9.

82 Narita K, Suda M, Takei Y, Aoyama Y, Majima T, Kameyama M, et al. Volume reduction of ventromedial prefrontal cortex in bipolar II patients with rapid cycling : a voxel-based morphometric study. Prog Neuropsychopharmacol Biol Psychiatry 2011; 35(2): 439-45.

83 Bassett D. Borderline personality disorder and bipolar affective disorder. Spectra or spectre? A review. Aust N Z J Psychiatry 2012; 46(4): 327-39.

84 Zanarini MC, Frankenburg FR, Hennen J, Silk KR. The longitudinal course of borderline psychopathology: 6-year prospective follow-up of the phenomenology of borderline personality disorder. Am J Psychiatry 2003; 160: 274-83.

85 Arnone D, McKie S, Elliott R, Juhasz G, Thomas EJ, Downey D, et al. Statedependent changes in hippocampal grey matter in depression. Mol Psychiatry 2012; 18: 1265.

86 Wang W-Y, Yu J-T, Liu Y, Yin R-H, Wang H-F, Wang J, et al. Voxel-based metaanalysis of grey matter changes in Alzheimer's disease. Transl Neurodegener 2015; 4(1): 6. 